\title{
Human calreticulin can act as adjuvant in the maturation of antigen presenting cells
}

\author{
A Bajor*1, J Hinrichs ${ }^{1}$, C Figueiredo ${ }^{1}$, M Wittmann ${ }^{2}$, R Blasczyk ${ }^{1}$ and B Eiz- \\ Vesper ${ }^{1}$
}

Address: ${ }^{1}$ Institute for Transfusion Medicine, Hannover Medical School, Hannover, Germany and 2Department of Dermatology and Allergology, Hannover Medical School, Hannover, Germany

* Corresponding author

from 12th Joint Meeting of the Signal Transduction Society (STS). Signal Transduction: Receptors, Mediators and Genes

Weimar, Germany. 29-31 October 2008

Published: 26 February 2009

Cell Communication and Signaling 2009, 7(SuppI I):A27 doi:I0.1 186/I478-8IIX-7-SI-A27

This abstract is available from: http://www.biosignaling.com/content/7/SI/A27

(c) 2009 Bajor et al; licensee BioMed Central Ltd.

Calreticulin (CRT), an endoplasmic reticulum (ER) resident protein, is involved in critical cellular functions, such as protein folding and antigenic peptide cross presentation. Furthermore, this chaperone has been proposed to act as an adjuvant during the activation of dendritic cells (DCs) in vivo. We assessed human eukaryotically expressed CRT for its potential to induce NF-kappa B regulated maturation of monocyte-derived DCs. In order to facilitate eukaryotic expression procedures, we established and compared three different methods to express recombinant endotoxin-free CRT to be secreted in the supernatant of HEK 293 cells: (1.) the complete, unmodified CRT coding sequence was cloned into the pcDNA3.1V5/His vector (euCRT), (2.) the C-terminal ER-retrieval KDEL amino acid sequence was mutated into KDQL in order to disturb the endoplasmic retention and support the protein secretion (euCRT_KDQL) and (3.) a shRNA was designed to knock down the expression of aminoacyltRNA synthetase-interacting multifunctional protein-1 (AIMP-1), which is known to regulate protein retention in the ER. An efficient shRNA sequence specific to AIMP-1 transcripts was delivered to HEK 293 cells, which were afterwards transfected with the CRT-expressing vector (euCRT). No relevant differences between these different approaches were observed in regard to mRNA levels of the transfected CRT determined by Real Time RT-PCR as well as protein expression levels of CRT determined by V5/HIS ELISA in the cell culture supernatants. Thus, for large scale expression of CRT the first strategy with the unmodified
CRT sequence (euCRT) was chosen. The functional capability of the expressed calreticulin to induce maturation of DCs was tested. By flow cytometry the translocation of NF-kappa B into the nuclei of the monocytes after stimulation with the recombinant CRT could be demonstrated. Using low-dose CRT $(10 \mu \mathrm{g} / \mathrm{ml})$ the phenoptype of the immature DCs changed to a more matured one, as indicated by an increased surface expression of CD40, CD86, CD83. In summary, our first data indicate, that this recombinant CRT can act as an adjuvant for in vitro maturation of DCs and therefore has the potential to assist in $\mathrm{T}$ cell stimulation and expansion protocols. 